$\mathrm{PB}-11$

\title{
High Resolution Refinement of Wild Type Human Lysozyme
}

Arno Pähler ${ }^{1}$, Koji Inaka and Masaaki Matsushima

Protein Engineering Research Institute, 6-2-3, Furuedai, Suita, Osaka, 565

1 Present address:

Taisho Pharmaceutical Company, 403 Yoshinocho-1-chome, Ohmiya, Saitama, 330

The structure of wild type human lysozyme has been refined at $1.5 \AA$ resolution using molybdenum $\mathrm{K}_{\alpha}$ radiation data collection collected with a MacScience Imaging Plate System DIP-100. The use of Mo $\mathrm{K}_{\alpha}$ made it possible to extend the accessible data region to $1.45 \AA$ and to reduce absorption and radiation damage. The starting model for refinement was a structure refined at $1.8 \AA$ resolution [K.Inaka et al., JBC 226 (19), 12599-12601, 1991]. A modified version of the program PROLSQ was used for the refinement. No low resolution cut-off was applied to the collected data and the influence of bulk solvent on the calculated low-resolution data was taken into account by applying Babinet's principle and modifying $\mathrm{Fc}$ according to $\mathrm{Fc}=\mathrm{Fc} *\left(1-\mathrm{K}^{\prime *} \exp \left(-\mathrm{B}^{\prime *} \mathrm{~s}^{2}\right)\right)$. Values for $\mathrm{K}^{\prime}$ and $B^{\prime}$ could be obtained by a least squares fitting procedure and were $K^{\prime}=0.849$ and $B^{\prime}=347.3 \AA^{2}$.

The final $R$-factor for a model consisting of 1252 atoms (1029 protein atoms +220 water oxygens + 3 ions) is $R=14.5 \%$ for the 15,599 reflections measured between $29.6 \AA$ and $1.5 \AA$ ( $80.6 \%$ complete). Rms deviations of bond distances from ideality are $0.014 \AA$ and $\mathrm{ms}$ deviations for other restraints also compare favourably to their ideal values. All solvent atoms were treated as disordered, i.e. no restraints were applied for non-bonded contacts involving solvent, to allow for multiple occupancies of water sites. Three solvent molecules were identified as ions rather than water oxygens. Two chlorine atoms, separated by $6 \AA$, were located on the basis of their high residual density in difference Fourier maps, one potential sodium ion was identified by its coordination stereochemistry. These findings were confirmed by other studies involving $\mathrm{NaBr}$ (for $\mathrm{Cl}$ ) and $\mathrm{LiCl}$ (for $\mathrm{Na}$ ) as solvent. Although some of the remaining peaks in difference Fourier maps strongly suggest that they mark hydrogen positions, no hydrogens were included in the present model. Inclusion of hydrogens would have reduced the $\mathrm{R}$-factor further by about $1 \%$ according to test refinements.

Not only is the R-factor reported here significantly lower than the one reported for the structure deposited in the Protein Data Bank, but also is the stereochemistry greatly improved compared to that model, in particular with respect to atomic mobility. As has been found by others, the refinement showed that accurate modelling of the solvent structure is important for obtaining improved results. Further refinement with a version of PROLSQ, modified to model bulk solvent and static disorder in an improved way, is planned. 